\title{
Naphthenic Acid Corrosion of 20G under High Flow Rate Flushing
}

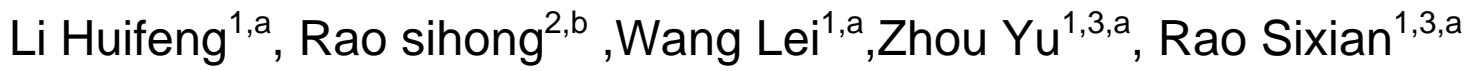 \\ 1. Anhui University of Technology, Maanshan, AnHui, 243032, China \\ 2. Huaihai Industrial Group Co., Ltd, changzhi, shanxi, P R China \\ 3. Hefei General Machinery Research Institute, Hefei, 230031, China

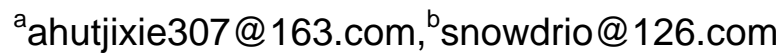

Keywords: 20G; Naphthenic acid corrosion; Flushing; Fluent

\begin{abstract}
Analysis of turbulence on the naphthenic acid corrosion specimens under scouring of $90 \mathrm{~m} / \mathrm{s}$ high flow rate fluid by Fluent indicated that maximum turbulence existed at the center of specimen, the turbulence in other zones decreased nonlinearly with the distance from the center. The average corrosion rate of 20G under the scouring of high flow rate corrosive media mainly depended on the corrosion temperature, the maximum average corrosion rate existed at the temperature of $320 \mathrm{C}$ was 2.5 times of the minimum average corrosion rate at temperature of $240 \mathrm{C}$. The influence of turbulence on naphthenic acid corrosion was shown in the local corrosion depth in different turbulence areas, experiments indicated that the ratio between maximum corrosion depth in high turbulence areas and average corrosion depth could reach 4 times or more at all experiment temperatures, so high turbulence could significantly increase local corrosion depth and lead to rapid thinning or perforation of pipelines.
\end{abstract}

\section{Introduction}

In recent years, due to the shortage and high costs of high quality oil, many refineries turned to refine high acid crude oil. Thus Naphthenic Acid Corrosion (NAC) of refinery equipments caused by naphthenic acids existed in high acid crude oil became serious. The field experience confirmed that in the zones that hindered the flow of fluid naphthenic acid corrosion was more serious ${ }^{[1-5]}$, particularly in the zones that high flow rate and strong turbulent existed . So research on naphthenic acid corrosion under high flow rate flushing was very important for corrosion rate prediction, evaluation and optimization of refinery equipment design.

In this paper, naphthenic acid corrosion of $20 \mathrm{G}$ under $90 \mathrm{~m} / \mathrm{s}$ flow flushing was investigated in order to determine the influence of flow rate, turbulence distribution on the naphthenic acid corrosion rate of 20G low carbon steel.

\section{Experimental method and equipment}

Experimental materials and experimental method. Low carbon steel 20G was selected as test material and the composition of $20 \mathrm{G}$ is listed in table 1.

Table1 Composition of 20G (mass fraction\%)

\begin{tabular}{ccccccccccc}
\hline content & $\mathrm{C}$ & $\mathrm{Si}$ & $\mathrm{Cu}$ & $\mathrm{Mn}$ & $\mathrm{P}$ & $\mathrm{S}$ & $\mathrm{Cr}$ & $\mathrm{Mo}$ & $\mathrm{Ni}$ & $\mathrm{Fe}$ \\
\hline $20 \mathrm{G}$ & 0.18 & 0.20 & 0.02 & 0.36 & $\leq 0.03$ & $\leq 0.03$ & - & - & 0.02 & $\mathrm{Bal}$ \\
\hline
\end{tabular}

The specimens were cut from sheets and machined to wafer structure with diameter of $14 \mathrm{~mm}$ and thickness of $5 \mathrm{~mm}$. The medium selected in experiments was high temperature heat conductive oil (high-purity Dibenzyl toluene) mixed with refined naphthenic acid, the TAN was $2.6 \mathrm{mg} \mathrm{KOH} / \mathrm{g}$. Finally the weight loss of each specimen was measured by an analytical balance. Threedimensional digital microscope $\mathrm{KH}-7700$ was used to scan the corrosion topography of specimens.

Laboratory equipment. Because NAC would not occur below 220C and naphthenic acids cracked above 400C, the temperature selected in the experiments were 240, 280, 320 and 360C. In order to investigate the influence of turbulence on NAC, The sketch of naphthenic acid corrosion 
simulation device was shown in Fig. $1^{[10]}$. The flow rate in the NAC simulation device could reach $400 \mathrm{~m} / \mathrm{s}$. Specific installation position and injection room diagram are shown in figure 2 and the specimens' surface flow flushing diagram is shown in figure 3.

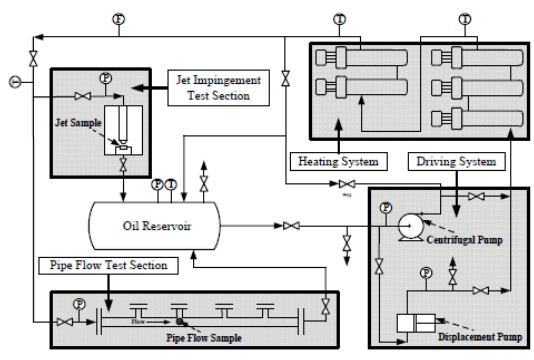

Fig.1 Sketch of NAC simulation device

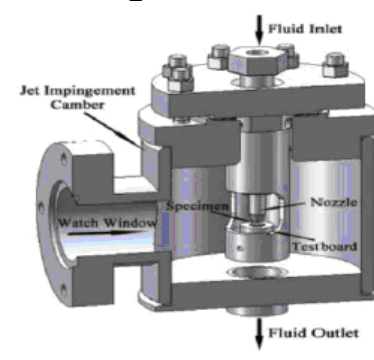

Fig.2 Sketch of Jet Impingement camber

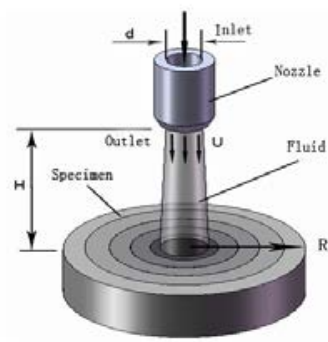

Fig. 3 Sketch of liquid impingement on the surface of specimens

The Computational Fluid Dynamics (CFD) is used to calculation of velocity distribution, turbulence intensity and wall shear stress of specimen surface under high speed flow flushing use .The software of Fluent was used to analyze to the turbulence on the specimen.

\section{Discussion of simulation results and test results}

Simulation results. For rational selection of spray nozzle height and the inlet velocity of experiments, Fluent simulation was made to test the flow rate and turbulence of the specimen surface under different velocity and different nozzle height $H$. The speed of flow field on the top of the injection specimens is shown in figure 4.It showed that the velocity field of the specimen when jet impacted includes free jet zone, impact zone and the radial wall jet zone. There was specific link between the flow field distribution and the nozzle height. The distribution of axial velocity under different height was shown in figure 5 . It showed that when the nozzle height $\mathrm{H}$ was $2 \mathrm{~mm}$, axial velocity decay quickly, only small region in the center of the specimen can keep high hit rate when flushed at a rate of $90 \mathrm{~m} / \mathrm{s}$, the velocity in the center of the distance decayed to half of its original rate away $\mathrm{R}=1 \mathrm{~mm}$. The flow outside the $\mathrm{R}=2 \mathrm{~mm}$ had been reduced to near zero, flow comes from nozzle rebounded after directly impact in the center of the specimen. When raised the nozzle height could be found that the injection rate of attenuation also reduced with the increase of height of nozzle. Considering the specimen used a diameter of $14 \mathrm{~mm}$, the height of nozzle controlled in 6 to $8 \mathrm{~mm}$ on the experiment, so the whole flushing rate on the surface of the specimen maintained a high level.

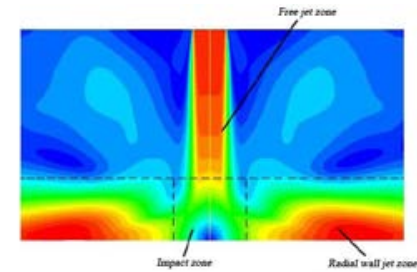

Fig. 4 the distribution of flow field under jet

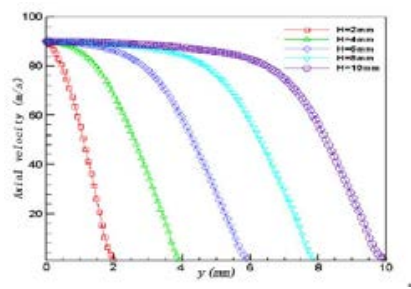

Fig. 5 flow rate distribution on the specimen under different height of nozzle

Fluent simulation results of specimen surface under different incident velocity turbulence showed that the turbulence intensity on the surface of the specimen related to jetted initial velocity $U$ and specimen from the center of radius $R$. When inlet velocity was $30 \mathrm{~m} / \mathrm{s}$, turbulence intensity distribution on the surface of the specimen was average between $2 \%$ and $3 \%$. When inlet velocity was $90 \mathrm{~m} / \mathrm{s}$, turbulence intensity distribution was at $6 \%$ to $9.5 \%$, and had the greatest turbulence intensity in the center of the circular specimen (figure 6). In order to obtain high local turbulence intensity, selecting $90 \mathrm{~m} / \mathrm{s}$ as inlet velocity was reasonable. 

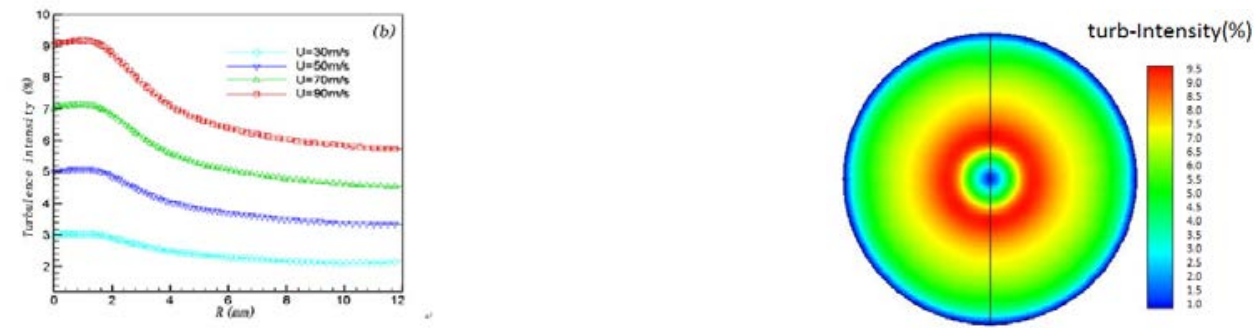

Fig.6 turbulence distribution on the specimen Fig.7 turbulence cloud chart on injection specimen

It showed from the figure 7 that the largest turbulence intensity was $9.5 \%$ and smallest turbulence intensity was slightly lower than $6 \%$ when inlet velocity was $90 \mathrm{~m} / \mathrm{s}$. The highest turbulence intensity on the surface of the specimen was not at the center of the specimen but occurred in the location of the center distance $1.52 \mathrm{~mm}$. The minimum turbulence intensity in the specimen occurred in the outside piece, but even at the edge the regional turbulence intensity was also as high as $6 \%$.

Effects on naphthenic acid corrosion rate of turbulent. Field experiences and the existing researches had confirmed the turbulent state had effect on naphthenic acid corrosion rate ${ }^{[6,7]}$, but the influence was not clear. At pipe flow mode, many groups of tests had got that parallel and vertical flow flushing had serious influence on average naphthenic acid corrosion rate and local corrosion depth under the velocity of $20 \mathrm{~m} / \mathrm{s}$. Results showed that the average of naphthenic acid corrosion rate under 320C was 2.0 to 3.0 times as an average of the corrosion rate under $240 \mathrm{C}$, but the influence was not significant. The average corrosion rate of $20 \mathrm{G}$ vertical flushing specimens and the parallel specimens had gap within $16 \%$. It was worth noting that the turbulence intensity had significant effects on the depth of local corrosion, the zone for local corrosion depth of 20G in turbulent intensity of $8 \%$ was 2.5 to 3 times in turbulence intensity $2 \%$ in the method of pipe flow.

Turbulence intensity and velocity were higher under injection ( the biggest turbulence intensity 9.5\%).To clear the impact on the naphthenic acid corrosion under the high speed flow flushing, corrosion experiments were tested at temperature of 240,280,320, 360C in the incident velocity of $90 \mathrm{~m} / \mathrm{s}$ and got the average corrosion rate and the local corrosion depth with the strong.

On the analysis of the corrosion tests showed that the average corrosion rate was $1.43 \mathrm{~mm} / \mathrm{a}$ at 240C , control the corrosion rate was relatively low. When the temperature elevated to $280 \mathrm{C}$, corrosion rate elevated to $2.45 \mathrm{~mm} / \mathrm{a}$. The corrosion rate was $3.63 \mathrm{~mm} / \mathrm{a}$ when temperature elevated to $320 \mathrm{C}$, naphthenic acid corrosion rate reached the maximum. When the temperature was up to $360 \mathrm{C}$, corrosion rate reduced to $2.16 \mathrm{~mm} / \mathrm{a}$ due to cracking of the naphthenic acid. At $320 \mathrm{C}$ and $240 \mathrm{C}$, the average corrosion rate ratio was about 2.5.And corrosion rate increased nearly two times when temperature raised every 55C in the empirical formula which was also consistent with the experimental results in the method of pipe flow.

The average corrosion rate can only reflected the temperature and turbulence intensity had influence on the overall corrosion rate. Naphthenic acid corrosion belonged to the typical chemical corrosion which was different from common electrochemical corrosion, and generally did not appear the electrochemical characteristics such as pitting corrosion but the groove morphology. It spread quickly in depth after the formation of groove .It led to perforation and leakage of the equipment. The evaluation of equipment service life was obviously unreasonable according to calculated the average corrosion rate. Existing researches showed that there was a certain correlation with the formation of the groove and turbulence state of the media ${ }^{[8,9]}$, so it needed to respectively analyze the local corrosion depth of strong, weak turbulence to determine the link. Due to turbulence intensity was no significant impact on the average corrosion rate, it was regarded as no turbulence or weak turbulence state corrosion rate. So it passed through the local corrosion depth and average corrosion depth ratio of the strong turbulent region to determine the influence of turbulence intensity. Due to the corrosion test of 8 hours, the average corrosion depth $d(\mu \mathrm{m})$ and the average corrosion rate $\mathrm{v}(\mathrm{mm} / \mathrm{a})$ can be conveyed by formula (1).

$$
d=\frac{v \cdot 8 h \cdot 10^{3}}{365(\text { day }) \times 24 h}=0.913 v
$$


The observation in different zones of the specimen on 3D morphology showed that corrosion depth of strong/weak turbulence zones existed significant differences. The maximum corrosion depth of the specimens was 4.8 microns (figure 8) in minimum turbulence zones (turbulence intensity about $6 \%$ ) at 320C, and the average corrosion depth was about $3.3 \mu \mathrm{m}$ according to the average corrosion rate and corrosion time. The ratio was about 1.45 . Low wall shear stress was lesser in turbulent flow, erosion corrosion was not significant. The corrosion product attached to a metal substrate was also inhibited to a certain extent. So the low turbulence intensity did not improve significantly the local corrosion depth. But the maximum corrosion depth at the middle of specimen in medium turbulence zones (turbulence intensity about $8 \%$ ) was $9.3 \mu \mathrm{m}$ (figure 9 ). It was 2.8 times of the average corrosion depth. The turbulence intensity had improved the local corrosion rate significantly. In high turbulence zones (turbulent intensity of 9.5\%), maximum corrosion depth was $12.8 \mu \mathrm{m}$ (figure 10).It was 3.88 times of the average corrosion depth. When turbulence intensity was close to $10 \%$ high turbulent state, wall shear stress was larger, the erosion effect was highlighted. At the same time, the corrosion products of metal surface were washed away quickly and metal matrix was bared. It made the chemical corrosion continue and the local corrosion depth was rapid expansion under the interaction between corrosion and chemical erosion.

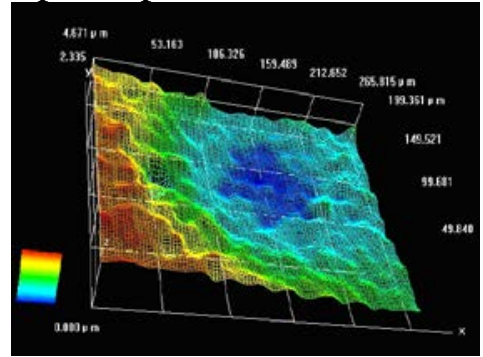

Fig.8 3D corrosion morphology Fig.9 3D corrosion morphology in low turbulence area at temperature of 320C

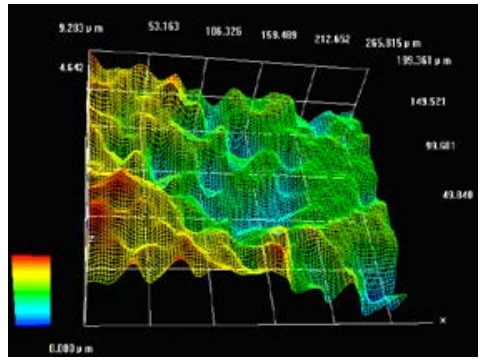

in medium turbulence area at temperature of 320C

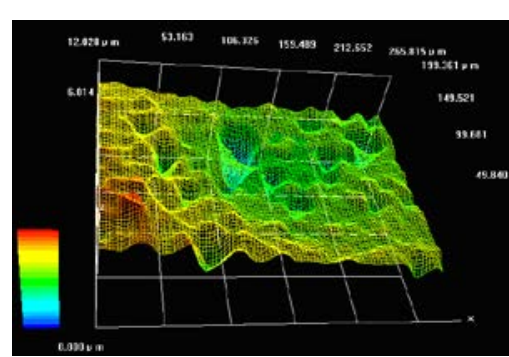

Fig.10 3D corrosion morphology in high turbulence area at temperature of 320C

Table2 Corrosion depth on different spots

\begin{tabular}{|c|c|c|c|c|c|c|}
\hline $\begin{array}{l}\text { Temperature } \\
\text { [C] }\end{array}$ & $\begin{array}{l}\text { Average } \\
\text { corrosion } \\
{[\mu \mathrm{m}]}\end{array}$ & depth & $\begin{array}{l}\text { Maximum } \\
\text { depth in } \\
\text { strong } \\
{[\mu \mathrm{m}]}\end{array}$ & $\begin{array}{l}\text { corrosion } \\
\text { region of } \\
\text { turbulence }\end{array}$ & $\begin{array}{l}\text { Maximum } \\
\text { depth in } \\
\text { weak } \\
{[\mu \mathrm{m}]}\end{array}$ & $\begin{array}{l}\text { corrosion } \\
\text { region of } \\
\text { turbulence }\end{array}$ \\
\hline 240 & & 21 & & 7.1 & & 2.9 \\
\hline 280 & & 24 & & 10.7 & & 4.1 \\
\hline 320 & & 32 & & 12.8 & & 4.8 \\
\hline 360 & & 97 & & 9.3 & & 4.0 \\
\hline
\end{tabular}

Other temperature corrosion data were shown in table 2. It could be seen from Table.2 that the ratio of maximum corrosion depth and the average corrosion depth are respectively 5.87, 4.78 and 4.72 under 240 C, 280 C, 360 Cin strong turbulent. And the ratio under 320C determined that local naphthenic acid corrosion depth in $10 \%$ high turbulence state was at least 4 times of the average corrosion depth. It can imagine if corrosion time is long enough, the region in high turbulence will form a macro corrosive groove that depth is larger. This phenomenon was consistent with the naphthenic acid corrosion groove that often appeared in the scene. High corrosion rate in high turbulence region led to refining component rapidly thinning even perforation.

\section{Conclusion}

(1) Turbulence intensity on specimen surface would decrease with increasing of distant from specimen center distance $\mathrm{R}$ under high-speed direct injection flow flushing. Highest turbulence intensity value existed in the center of the specimen which was about $9.5 \%$.

(2) The test results showed that specimens had the highest corrosion rate in strong turbulent zones, local corrosion depth was 4 times of the average corrosion depth in strong turbulent zones. 
(3) Due to existing significant difference of corrosion rate in the different turbulence intensity zones, local trench could be formatted in high turbulence intensity zones and rapidly expansion. Finally it led to a rapid reduction of the thickness of oil refining equipment wall and even perforation.

\section{Reference}

[1] Liang Chunlei, Chen Xuedong, Ai Zhibin, Overview of the mechanism of Naphthenic Acid Corrosion and Its Influencing Factors[J],Journal of pressure equipment and systems,2008,25(5): 30-36

[2] Gao Yanmin, Chen Jiajian, Lei Liangcai. The Status-quo of Research on Naphthenic Acid Corrosion and Protection[J]. Corrosion \& Protection in Petrochemical Industry,2000,17(2):6-12

[3] Chen Bifeng, Yang Qiming. Kinetics Analysis of Naphthenic Acid Corrosion of Alloy steels for Atmospheric and Vacuum Equipment[J],Corrosion Science and Protection Technology, 2007,19(1): 74-76

[4] SLAVCHEVAE,SHONEB,TURBULLA. Review of naphthenic acid corrosion in oil refining[J], British Corrosion Journal,1999,34(2):125-131

[5] NUGENT M J,DOBIS J D. Experience with Naphthenic Acid Corrosion in low TAN Crudes[C].Corrosion98,Houston,NACE International, 1998: 577-586

[6] GROYMANA, BRODSKYN, PENNERJ. etc. Low Temperature Naphthenic Acid Corrosion Study[A],Corrosion, Houston, NACE International, 2007: 569-578

[7] CRAIGHL. Temperature and Velocity Effects in Naphthenic Acid Corrosion [A].Corrosion 96,Houston, NACE International,1996:603-613

[8] WU Xinqiang, JING Heming, ZHENG Yugui. etc. Erosion corrosion of various oil refining materials in naphthenic acid[J],wear,2004,256:133-144

[9] OMAR YEPEZ.On the Chemical Reaction between Carboxylic Acids and Iron, Including Special case of Naphthenic Acid. Fuel [J],2007, 86:1162-1168

[10] Xu Peng, Chen Xuedong, Qin Zongchuan. etc. High temperature and high-flow-rate testing device on naphthenic acid corrosion and test procedure[P]. China,CN101477027,2009 